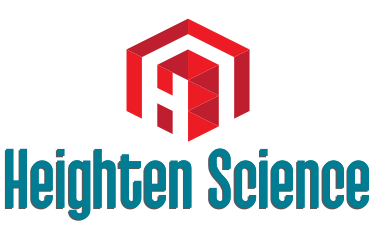

P U B L I C I T I O N S Corporation

\title{
Trypanosoma dionisii as an experimental model to study anti-Trypanosoma cruzi drugs: A comparative analysis with benznidazole, posaconazole and amiodarone
}

\author{
Barrias ES ${ }^{1}$, Borges TR 1 and De Souza $\mathrm{W}^{2,3^{*}}$ \\ ${ }^{1}$ National Institute of Metrology, Quality and Technology (Inmetro), Direction of Metrology \\ Applied to Life Sciences - Laboratory of Microscopy Applied to Life Sciences - Av. Nossa \\ Senhora das Graças, 50/27, Xerém, Duque de Caxias, Rio de Janeiro 20220-050, Brazil \\ ${ }^{2}$ Cellular Ultrasound Laboratory Hertha Meyer, Carlos Chagas Filho Institute of Biophysics, CCS, \\ UFRJ, Carlos Chagas Av. 373, CCS, City University, RJ 21941-902, Brazil \\ ${ }^{3}$ National Institute of Science and Technology in Structural Biology and Bioimaging and \\ National Center for Bioimaging-CENABIO, Federal University of Rio de Janeiro, City University, \\ RJ 21941-902, Brazil
}

\begin{abstract}
*Address for Correspondence: De Souza W Cellular Ultrasound Laboratory Hertha Meyer, Carlos Chagas Filho Institute of Biophysics, CCS, UFRJ, Carlos Chagas Av. 373, CCS, City University, RJ 21941-902, Brazil, Tel: +552139386480; Email: wsouza@inmetro.gov.br; wsouza@biof.ufrj.br
\end{abstract}

Submitted: 04 October 2018

Approved: 16 October 2018

Published: 17 October 2018

Copyright: @ 2018 Barrias ES, et al. This is an open access article distributed under the Creative Commons Attribution License, which permits unrestricted use, distribution, and reproduction in any medium, provided the original work is properly cited

Keywords: T. cruzi; T. dionisii; Chemotherapy

Check for updates

\section{Abstract}

Chagas disease is a public health problem in Latin America and its treatment is based on the use of benznidazole or nifurtimox compounds. Both present problems such as resistance, inefficiency in chronic infection and cytotoxic effects. New compounds such as posaconazole and amiodarone have been tested against $T$. cruzi and shown to be effective. In addition, new molecules have been synthesized and tested against $T$. cruzi. Because this protozoan is highly pathogenic, even with a number of cases of accidental laboratory infections, few laboratories located outside Latin America are authorized to work with its infective developmental stages. On the other hand, Trypanosoma dionisii is a non-pathogenic protozoan phylogenetically related to $T$. cruzi and that shares similar strategies to complete its life cycle in mammalian cells in vitro. Here, we describe a comparative analysis of the sensitivity of both parasites to benznidazole, posoconazole and amiodarone. We also analyzed the morphological effects of these compounds on both Trypanosoma species using electron microscopy. Our results show that $T$. dionisii is more sensitive to the compounds tested than $T$. cruzi. They also support a previous suggestion that it may constitute an excellent model for large scale screening of compounds against $T$. cruzi.

\section{Introduction}

Chagas disease is recognized by the World Health Organization (WHO) as one of the world's 13 most neglected tropical diseases [1]. It is a chronic, systemic and endemic disease caused by Trypanosoma cruzi - an obligatory intracellular parasite belonging to the Kinetoplastid order. The disease affects approximately 16 million people in Latin America [2] and is the major parasitic disease burden on the American continent [3,4]. Chagas disease is found worldwide due to international immigration [2]. The parasite life cycle encloses vertebrate and invertebrate hosts where different developmental stages are found. These include the proliferative amastigote and epimastigote forms, which divide within the vertebrate host cell or in the intestine of the invertebrate host, respectively. Trypomastigote form is released from the host cells and found in the intercellular space and the bloodstream or in the posterior regions of the intestine of 
the invertebrate hosts [5,6]. While traditionally recognized as a disease transmitted to humans via the insect vector, more recent data has shown an increase in infection by a new mechanism of oral transmission due to the ingestion of contaminated fruit juices in the Amazonian region [7,8]. This has led to the observation of new cases of acute Chagas disease [9]. In addition, millions of patients with chronic Chagas disease wait for better drugs since the first choice compounds present a poor efficacy in treating chronic stage disease and cause innumerable collateral effects [10].

Current treatment of Chagas' disease uses nitroheterocycles including nifurtimox (Nfx) and benznidazole (Bzn). Their efficacy during the chronic phase is still controversial with poor indices of parasitological cure [11,12]. Despite the large number of people infected, less than $1 \%$ of new therapeutic drugs commercialized in the last 30 years have been directed to the treatment of tropical diseases [13]. In this context, there is an urgent need of new alternatives for the treatment of Chagas disease. One significant advance was recently reported showing that GNF6702, an inhibitor of parasite proteasome, is highly efficient against T. cruzi in vitro as well as in vivo [14]. WHO has defined target profiles for the production of novel compounds including two key elements: efficiency following oral application to patients with acute, indeterminate and chronic phases of the disease and a treatment time under 60 days. The ideal drug should be stable and inexpensive. Additional requirements are that the molecule be highly specific against the parasite with low or no toxicity to the patient [1].

Relatively few laboratories outside Latin America are able to screen and evaluate new compounds for Chagas disease therapy using the trypomastigote and amastigote infective stages. In several countries, legislation makes it difficult to work with this pathogenic protozoan. Indeed, several lethal cases of T. cruzi infection have been reported due to accidental exposure in research laboratories $[15,16]$. Indeed, laboratory accidents during manipulation of T.cruzi are relatively frequently, although usually not reported to health authorities. Several laboratories even start treatment of such cases using benznidazole. Thus, many laboratories screen anti-T. cruzi compounds using the noninfectious epimastigote form maintained in axenic cultures. However, large libraries of potential therapeutic compounds are now available and should be screened using the best model possible.

There is a close phylogenetic relationship between T. cruzi and T. dionisii, and several authors have suggested that these parasites use similar strategies to complete their life cycles $[17,18]$. T. dionisii is a non-pathogenic trypanosomatid isolated from bats that can infect mammalian cells in vitro by exploiting the same routes of entry and maintenance of T. cruzi infection [19]. Both species share antigenic components and epitopes that are recognized by monoclonal antibodies against $T$. cruzi $[19,20]$. The data suggest that $T$. dionisii is a potential experimental model for initial screening and further biochemical studies related to the development of new drugs for the treatment of Chagas disease, as previously suggested by Gutteridge group in 1977 [21].

Based on this assumption, we performed a comparative analysis of some compounds previously described as highly effective against $T$. cruzi using $T$. dionisii. We used epimastigotes and intracellular amastigotes of both species that were treated with benznidazole as well as amiodarone and pozaconazole. Benznidazole is the compound presently used to treat Chagas disease in Brazil [22]. Amiodarone was selected due to the fact that it is routinely used to treat heart arrythimia in patients with the cardiac form of Chagas disease [23,24] and has been shown recently to inhibit the sterol biosynthesis pathway and kill T. cruzi [25]. Posoconazole has also been shown to kill T. cruzi both in vitro and in vivo and is being tested in patients with Chagas disease [26-29]. The results validate the non-pathogenic trypanosomatid T. dionisii as a model for the study of new anti T. cruzi drugs and it is now being used in our platform of screening of compounds against $T$. cruzi that is supported by the Ministry of Health of Brazil, the National Research Council of Brazil (CNPq) and the Carlos Chagas Filho Foundation that supports research activity in Rio de Janeiro (FAPERJ). 


\section{Materials and Methods}

\section{Parasite strain and cell cultures}

T. dionisii TCC211 strain was isolated from a bat and maintained in the USP Trypanosomatids Bank. It was kindly provided by Dr. Martha Teixeira and Dr. Erney Camargo (Universidade de São Paulo - USP). T. dionisii epimastigotes were cultivated in a TC100 medium (Cultilab) supplemented with 10\% fetal bovine serum (FBS-Invitrogen) at $28^{\circ} \mathrm{C}$ for $4-5$ days or until it reached the log phase of growth as described in Maeda et al. [18]. Epimastigotes of the T. cruzi (Y strain) were cultivated in LIT medium [30] supplemented with 10\% FBS (Invitrogen). The cultures were maintained at $28^{\circ} \mathrm{C}$ for $4-5$ days or until they reached the log phase of growth. New cultures were made after 4 days from an initial inocculum of $2 \times 10^{6}$ parasites $/ \mathrm{mL}$. $T$. dionisii (TCC211) and T. cruzi (TCY) trypomastigotes were maintained in LLC- $\mathrm{MK}_{2}$ epithelial cells of animal origin obtained from the kidneys of Rhesus monkeys (Macaca mulatta -ATCC, CCL-7). The epithelial cells were grown in culture bottles (Biosystems; $25 \mathrm{~cm}^{3}$ ) in RPMI 1640 medium (Thermo) supplemented with 5\% FBS (Cultilab) at 37 $\mathrm{C}$ under an atmosphere of $5 \% \mathrm{CO}_{2}$. After confluence, the epithelial cells were treated with a trypsin-versene mixture (Sigma-Aldrich) (0.2 and $0.002 \%$, respectively), and the loose cells were collected in RPMI medium and seeded in new bottles $\left(2 \times 10^{5}\right.$ cells $/$ $\mathrm{ml}$ ). After 24 hours, cells were infected with $2 \times 10^{6}$ trypomastigotes (TCC211 or TCY strain). Five to seven days after infection, the trypomastigotes were collected from the culture supernatant.

Resident peritoneal macrophages were obtained from Swiss mice according to the Ethics Committee for Animal Experimentation of the Health Sciences Centre, Federal University of Rio de Janeiro (Brazil) under protocol number IBBCF096/097. The macrophages were collected using Hank's solution, plated on $13 \mathrm{~mm}$ round glass coverslips and allowed to adhere for 45 minutes at $37^{\circ} \mathrm{C}$ in a $5 \% \mathrm{CO}_{2}$ atmosphere.

Subsequently, non-adhered cells were removed by washing with Hank's solution and RPMI 1640 medium with 10\% FBS. The cells were maintained in culture for 24 hours at $37^{\circ} \mathrm{C}$ in $5 \% \mathrm{CO}_{2}$ before experiments.

\section{Chemicals and drug solutions}

We used benzdinazole (Lafepe, Brazil), pozaconazole (ScheringPlough Research Institute) and amiodarone hydracloride (Teva Farm). Stock solutions of all compounds were prepared in dimethyl sulphoxide (DMSO) (Merck, Darmstadt, Germany) with the final concentration of DMSO in the experiments never exceeding $0.05 \%$.

\section{Effect of compounds on T. cruzi and T. dionisii}

The susceptibility of $T$. dionisii (and T. cruzi) to beznidazole, amiodarone and posaconazole was evaluated using parasite proliferation curves in the absence or presence of drugs alone or in combination. Epimastigote cultures were initiated at a cell density of $1.0 \times 10^{6}$ cells $/ \mathrm{ml}$ and benznidazole, posaconazole and amiodarone were added at different concentrations after $24 \mathrm{~h}$ of growth. Cell numbers were evaluated daily in a Neubauer chamber during 96 h of growth.

To evaluate the effects of compounds on T. dionisii (and T. cruzi) intracellular amastigotes, macrophages were infected as described previously and incubated with different concentrations of the compounds after $24 \mathrm{~h}$ of infection. Next, cultures were labeled with Hoescht $3348(5 \mu \mathrm{g} / \mathrm{ml})$ and WGA-Fitc $(1 \mu \mathrm{g} / \mathrm{ml})$. Fresh media with drugs was added daily for 3 days ( $72 \mathrm{~h}$ of treatment). The number of intracellular amastigotes and macrophages (infected or not) were counted by nucleus and kinetoplast staining (macrophages and parasites) with high content analysis equipment (Incell $2000 \mathrm{GE}$ Healthcare) equipped with $20 x$ magnification (N.A $=0.45)$. Image analysis used Incell Investigator software (module organelles). Association indices (the mean number of 
parasites internalized multiplied by the percentage of infected macrophages divided by the total number of macrophages) were determined and used as a parameter to calculate the percentage of infection for each condition. The concentration that inhibited $50 \%$ of growth (IC50s) was calculated using SigmaPlot ${ }^{\circledR}$ v.10 157 (Systat Software Inc., San Jose, CA, USA). At least three independent experiments were performed for each condition.

\section{Electron microscopy}

Scanning electron microscopy: The epimastigotes were cultivated as described above. After each treatment, the cells were washed and fixed in a solution containing $2.5 \%$ grade I glutaraldehyde (TedPella, Redding, CA, USA) in $0.1 \mathrm{M}$ cacodylate buffer, $\mathrm{pH} 7.2$ for 30 minutes to 1 hour. They were then postfixed with $1 \% \mathrm{OsO}_{4}$ in $0.1 \mathrm{M}$ cacodylate buffer, pH 7.2, dehydrated in an ethanol series (30, 50, 70, 90 and 100\%), critical point-dried in a Baltec CPD 030 apparatus and mounted on specimen stubs. The samples were ion sputtered to avoid charge effect with a $10 \mathrm{~nm}$ gold layer and observed with a Quanta FEI-FEG scanning electron microscopy operating at $5 \mathrm{kV}$.

Transmission electron microscopy: Epimastigotes and trypomastigotes were cultivated as previously described. Intracellular amastigotes were allowed to grow in $60 \mathrm{~mm}^{2}$ Petri dishes (TPP, Trasadingen, Switzerland). After the experimental procedure, the cells were washed, fixed, and post-fixed as described above. Next, the cells were dehydrated in increasing concentrations of acetone and embedded in Epon. Ultrathin sections were stained with uranyl acetate and lead citrate and observed under a Jeol 1200EX or Zeiss EM900 transmission electron microscope.

\section{Immunofluorescence microscopy analysis}

For immunofluorescence microscopy assays, macrophages were infected for 24 h, treated with IC 50 posaconazole, and incubated for $96 \mathrm{~h}$ at $37^{\circ} \mathrm{C}$. Samples were fixed in $4 \%$ formaldehyde (30 min), permeabilized with $0.1 \%$ Saponin in $3 \%$ bovine serum albumin (BSA) in phosphate buffered saline (PBS) (pH 8.0) for $30 \mathrm{~min}$ at room temperature, and blocked with the same solution for $15 \mathrm{~min}$. The cells were incubated overnight with rabbit anti-LC3B antibody (Sigma Chemical Co., St Louis, MO, USA) at a dilution of 1:100. They were then rinsed and incubated for $45 \mathrm{~min}$ at room temperature with Alexa Fluor® 456-conjugated goat anti-rabbit IgG (Molecular Probes, Carlsbad, CA, USA) diluted to 1:400. The cells were subsequently rinsed in 3\% BSA/PBS (pH 8.0) and incubated with Hoechst dye (Molecular Probes) to label the nuclei. Coverslips were mounted and observed on a Zeiss Axioplan fluorescence microscope (Carl Zeiss GmbH, 215 Jena, Germany).

\section{Ethical guidelines}

This study was approved by the Ethics Committee of the Carlos Chagas Filho Institute (Protocol no. IBCCF106). All animals received humane care in compliance with the Principles of Laboratory Animal Care formulated by the National Society for Medical Research and the 'Guide for the care and use of laboratory animals' prepared by the USA National Academy of Sciences (Washington, DC).

\section{Results}

Antiproliferative effects of benznidazole, amiodarone, and posaconazole against $T$. dionisii and $T$. cruzi

Parasites (TCC211 and TCY) were treated with increasing concentrations of compounds $(0.5,1,3,5$ and $10 \mu \mathrm{M}$ to pozaconazole or amiodarone treatment and 0.5 , $1,5,10$ and $20 \mu \mathrm{M}$ to benznidazole treatment) for $96 \mathrm{~h}$ at $28^{\circ} \mathrm{C}$ to determine the effect on $T$. dionisii epimastigote growth and compare the effects with T. cruzi. All compounds were effective against T. dionisii and T. cruzi epimastigotes, which caused concentration and time dependent inhibition on growth (Figure 1). This figure also show that $T$. dionisii epimastigotes were around ten times more sensitive to the compounds than $T$. 
cruzi epimastigotes. After 72 hours of benznidazole treatment, the IC50 values (Figure 1A-B) were approximately $1.3 \mu \mathrm{M}$ and $10 \mu \mathrm{M}$ for T. dionisii and T. cruzi epimastigotes, respectively. In relation to pozaconazole treatment, IC50 values were $2.3 \mathrm{nM}(T$. dionisii) and $25 \mu \mathrm{M}$ (T. cruzi) (Figure 1C-D). Treatment with amiodarone showed IC50 values of $1 \mu \mathrm{M}$ for $T$. dionisii and $8 \mu \mathrm{M}$ for T. cruzi (Figure 1D-E). These observations show that $T$. dionisii epimastigotes were more sensitive to the three compounds than T. cruzi after 96 hours of incubation.

When treatment was done in intracellular amastigotes (the clinically relevant form of the parasite), the drug effects were more pronounced (Figure 2). The compounds showed a drastic reduction in intracellular amastigotes of both species also in a dose dependent manner. The effect was higher in T. dionisii than in T. cruzi. In terms of intracellular amastigotes, treatments with benznidazole (Figure $2 \mathrm{~A}$ ) results in an IC50 value of $2 \mu \mathrm{M}$ for $T$. dionisii and $5 \mu \mathrm{M}$ for T. cruzi. The IC50 for posaconazole treatment after 96 hours was $0.25 \mathrm{nM}$ for T. dionisii and $1 \mathrm{nM}$ for T. cruzi with posaconazole (Figure 2B). When amastigotes were treated with amiodarone (Figure 2C) an IC50 of 5 $\mu \mathrm{M}$ (T. cruzi) and $3 \mu \mathrm{M}$ (T. dionisii).
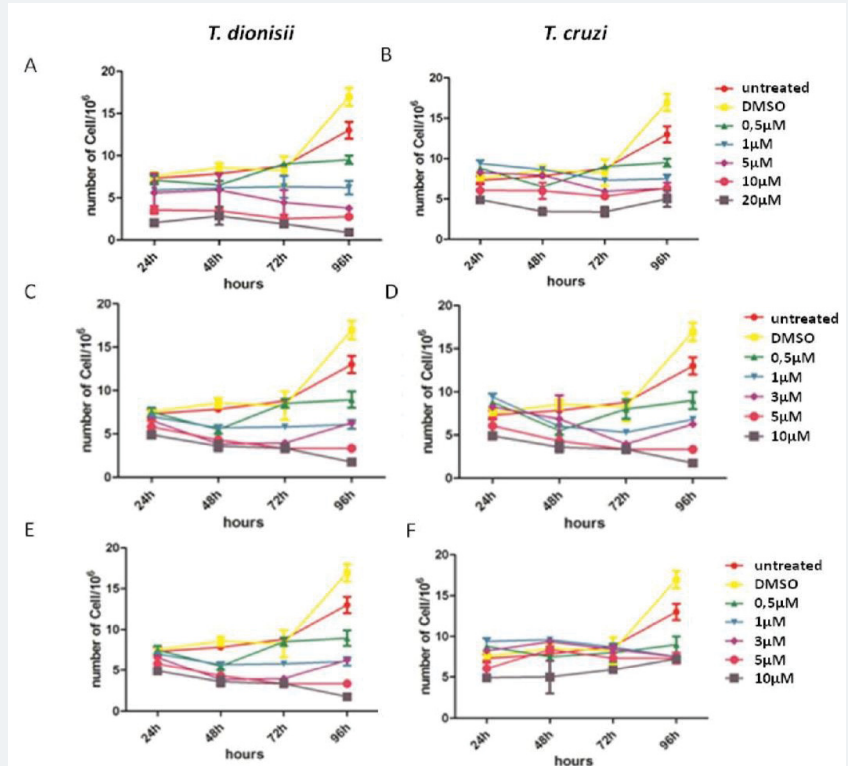

Figure 1: Trypanosoma cruzi and Trypanosoma dionisi epimastigotes incubated in the presence of benznidazole, posaconzole and amiodarone inhibit growth, but the inhibition occurs at lower concentrations in T. dionisii. A-F: Growth curve of epimastigote forms of $T$. cruzi and T. dionisii treated with $0.5,1,5,10$ and $20 \mu \mathrm{M}$ of Bzn (A-B), 0.5, 1, 3, 5 and $10 \mu \mathrm{M}$ of posaconazole (C-D) and $0.5,1,3,5$ and $10 \mu \mathrm{M}$ of amiodarone (E-F). The drugs were added with $24 \mathrm{~h}$ of culture. The number of parasites was determined every day over three days using a Neubauer chamber and a light microscope (objective $40 \times, N A=0.9$, phase contrast). The results are expressed as the mean of three independent experiments.

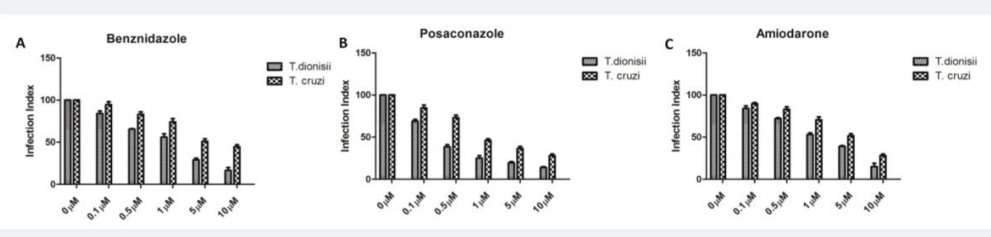

Figure 2: Treatment of peritoneal macrophages infected with amastigotes of $T$. cruzi or $T$. dionisii with benznidazole. (A), posaconazole (B) and amiodarone (C) inhibits amastigotes' multiplication at different concentrations depending on the Trypanosoma species used. The drug was added in the macrophage cultures after $24 \mathrm{~h}$ of interaction, when most of the cells were infected. Every day, the association indices were determined (mean number of parasites internalized multiplied by the percentage of infected macrophages divided by the total number of macrophages). The results are expressed as the mean of three independent experiments. 
Morphological analysis of $T$. dionisii epimastigotes and amastigotes treated with posaconazole and amiodarone

In a previous manuscript, we described the fine structure of control and drugtreated epimastigote and amastigote forms of T. cruzi [28]. T. dionisii epimastigotes treated with $1 \mu \mathrm{M}$ posaconazole or with $5 \mu \mathrm{M}$ amiodarone for 72 hours were analyzed by scanning electron microscopy (SEM) and transmission electron microscopy (TEM). SEM analysis of untreated $T$. dionisii epimastigotes revealed an elongated shape with a smooth cell surface (Figure 3A). After treatment with posaconazole, the flagellum becames shortened (Figure 3B - arrows). Treatment with amiodarone promoted an epimastigotes changed from their typical elongated shape to a spherical shape (Figure 3C - arrow), where the presence of more than one flagellum in the same twisted and rounded cell body can be observed (Figure 3C - arrowhead). By TEM, after treatment with $1 \mu \mathrm{M}$ posaconazole, there was a drastic alteration in the Golgi complex, i.e., disorganization of the cisternae leading to the appearance of dilated and empty spaces (Figure 4B), morphology not observed in untreated epimastigotes (Figure 4A - GC). The formation of vesicles in the plasma membrane of the parasite (Figure $4 \mathrm{C}$ - arrow)as well as the presence of endoplasmic reticulum involving intracellular structures suggesting an autophagic process (Figure 4C - bold arrow) after treatment with $5 \mu \mathrm{M}$ amiodarone. The subpelicular microtubules did not present any type of abnormality after treatment (Figure 4C - arrowhead). Ultrastructural analysis of intracellular amastigotes treated with $0,25 \mathrm{nM}$ posaconazole (Figure $5 \mathrm{~B}$ ) and $3 \mu \mathrm{M}$ amiodarone (Figure $5 \mathrm{C}$ ) by TEM demonstrated alterations in the Golgi complex including mischaracterization of its lamellae (Figure 5B-C arrows) when compared with untreated amastigotes. When intracelullar amastigotes were treated with posaconazole also demostrated a presence of internal membranes, suggesting the begin of authophagic process (Figure 5D).

\section{Cell death by autophagy in intracellular amastigotes}

Infected macrophages were analyzed by immunofluorescence microscopy using an anti-LC3B antibody to identify autophagic structures and elucidate the mechanism of cell death in intracellular amastigotes treated with posaconazole at IC 50 concentrations (Figure 6). Approximately 30\% of the macrophages infected with amastigotes were strongly labeled after treatment with the compounds. No labeling was observed in untreated amastigotes suggesting that the treatment with posaconazole induced amastigote authophagy (Figure 6A, B and C) as previously reported for T. cruzi [28].

\section{Discussion}

It is well recognized that it is very important to increase research in Chagas disease treatment since it is still prevalent in several countries. We show that the use of Trypanosoma dionisii, a member of the Trypanosoma genus and Schyzotrypanum subgenus, may constitute an alternative model for drug screening and further biochemical studies on the mechanism of Chagas therapy. Since the classical studies carried out by Baker and Selden [31], bloodstream trypomastigotes have been isolated from bats and shown to infect mammalian cells in vitro. They transform into amastigote forms that proliferate within the host cells and subsequently transform back in trypomastigotes that are released into the extracellular medium and infect new cells.

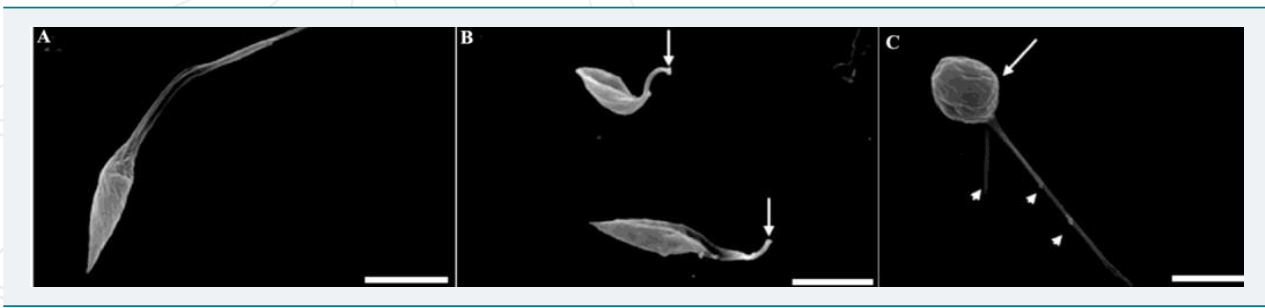

Figure 3: Posaconazole- and amiodarone-induced surface alterations in T. dionisii epimastigotes observed using SEM. Untreated epimastigotes (A) display usual morphologies and surface topologies. Epimastigotes treated with $1 \mu \mathrm{M}$ posaconazole during 72 hours (B) showed a cell body contortion while treatment with $5 \mu \mathrm{M}$ amiodarone demonstrated a dramatic reduction in flagellum length (C). Bars $=1 \mu \mathrm{m}$. 


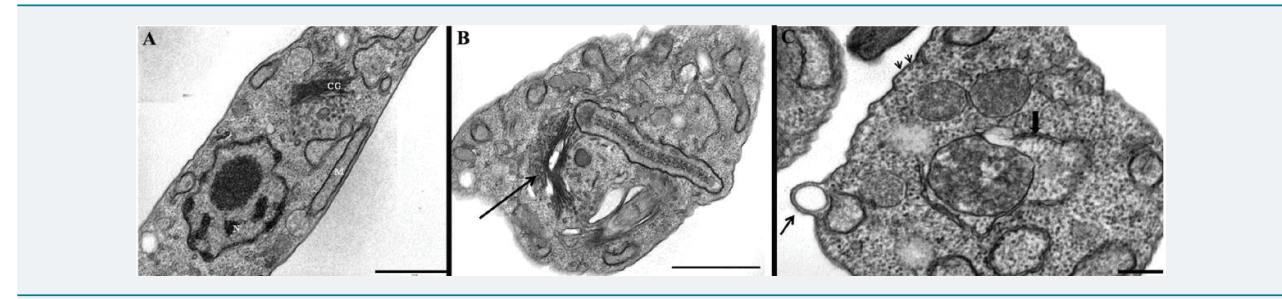

Figure 4: Transmission electron micrographs of $T$. dionisii epimastigotes treated with posaconazole and amiodarone (B-D). (A) Control cell with normal morphology $(N$, nucleus; $k$, kinetoplast; $F$, flagellum). (B) Epimastigotes treated with $1 \mathrm{nM}$ posaconazole showed an intense disorganization of Golgi complex (asterisks). (C-D) Amiodarone treatment $(0.5 \mu \mathrm{M})$ causes mitochondrial swelling $(\mathrm{m})$ and membrane blebs (b) at the parasite flagellum after 48 hours.

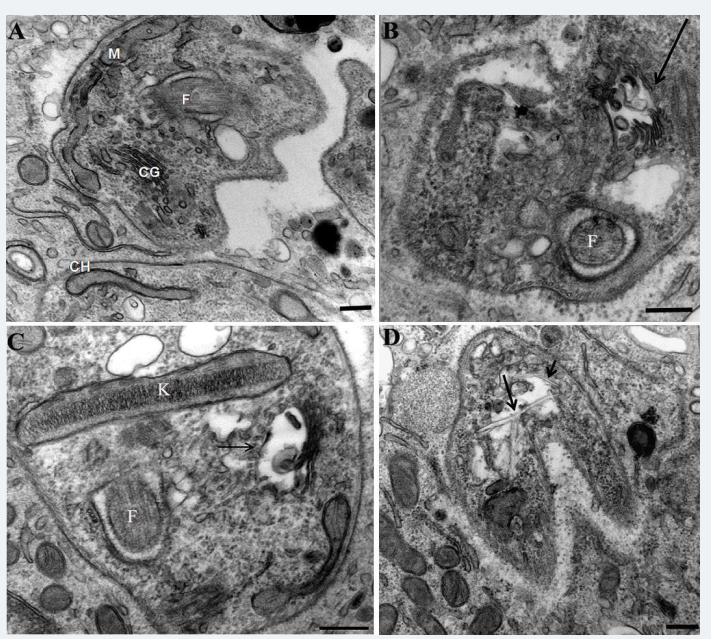

Figure 5: Transmission electron micrographs of intracellular amastigotes after 24 hours of treatment with posaconazole and amiodarone. (A) Untreated amastigotes inside peritoneal macrophages display a normal morphology. N, nucleus; k, kinetoplast; F, flagellum; G, Golgi). (B) After 24 hours of treatment with $1 \mathrm{nM}$ posaconazole, amastigotes appear with autophagic structures (arrow) and dilated Golgi complex lamellae (asterisks). (C-D) Treatment of intracellular amastigotes with 0.5 $\mu \mathrm{M}$ amiodarone caused alterations in the kinetoplast ( $\mathrm{k}$ ), with membrane detachment (arrow) and appearance of internal vesicles (arrowhead).

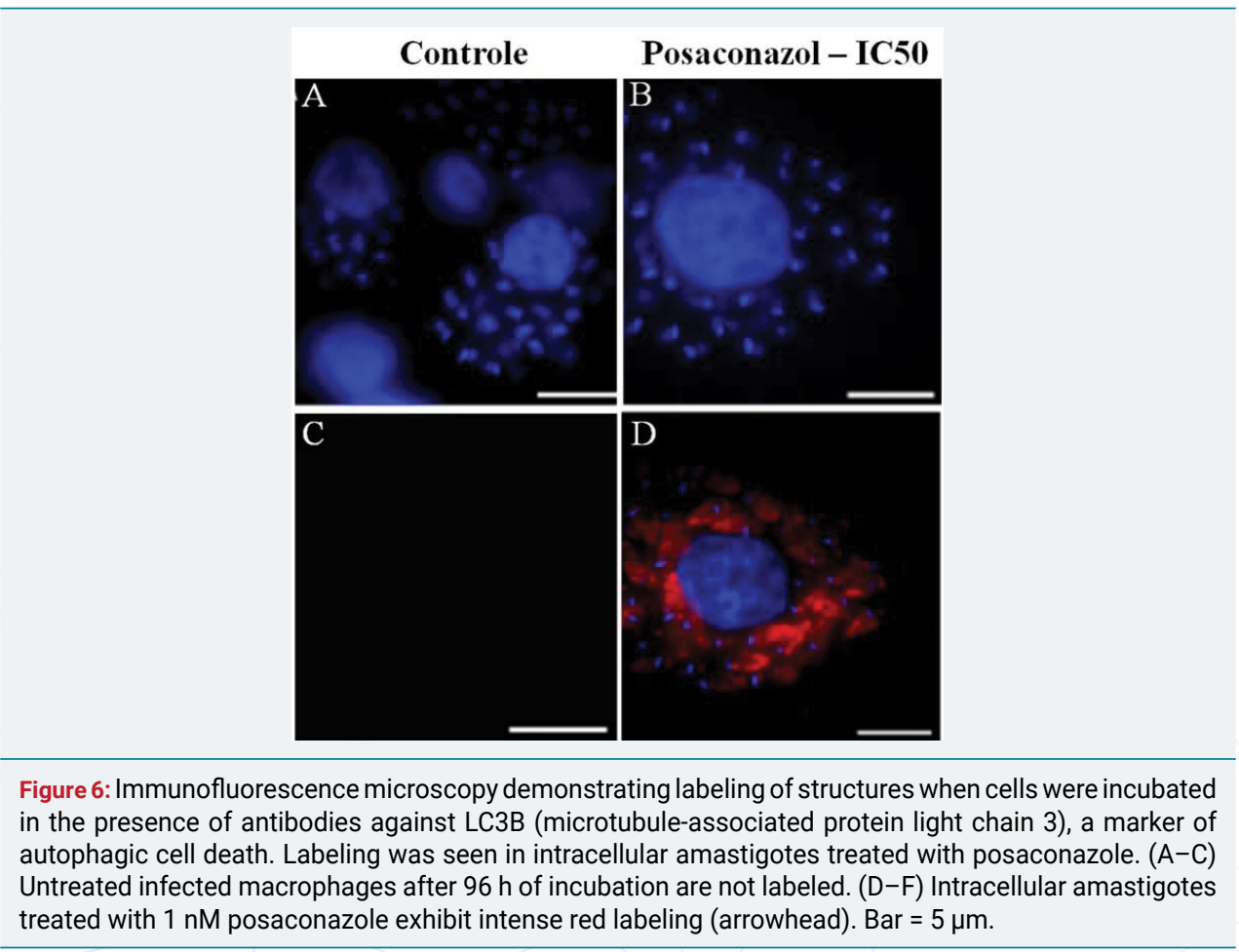


When the IC 50 results obtained with the $T$. dionisii treatment are compared with results reported for T. cruzi, using amiodarone or posaconazole, we note that there is a reduction of about 10 fold. Both compounds are capable to inhibit sterol biosystems. Sterols are crucial components of eukaryotic cells since its contributes to the stability, permeability and fluidity of the membrane. The sterol produced by protozoan is the ergosterol, while mammalians produce cholesterol. Trypanosoma cruzi depends on de-novo ergosterol production for its survival in all the stages of its life cycle [32]. The dependence of endogenous production makes T. cruzi very sensitive to inhibitors of this pathway [33]. There are no studies describing the biosynthesis of steroids in Trypanosoma dionisii and probably the greater susceptibility to the inhibitor treatments of this pathway is an indication that this species is even more dependent on an endogenous production when compared to T. cruzi. In relation to amiodarone, this compound is widely known as intracelullar calcium disruptor [34]. Recently, posaconazole by itself is able to produce an increase in the intracelullar parasite calcium concentration [34]. Maeda and colleagues [18] had already demonstrated that infection of a host cell by $T$. dionisii occurs by calcium dependent mechanisms and its dependence also could be related to treatment susceptibility. We also show that the epimastigote and amastigote forms of $T$. dionisii are sensitive to the benznidazole, a drug routinely used to treat Chagas disease in Brazil. Benznidazole is thought to be reduced to various electrophilic metabolites by nitroreductases present in Trypanosoma cruzi [35]. These metabolites likely bind to proteins, lipids, DNA, and RNA resulting in damage to these macromolecules. Benznidazole has been found to increase trypanosomal death through interferon- $\gamma$ which is likely present in increased amounts due to inflammation caused by macromolecule damage [36]. DNA in parasites affected by benznidazole has been found to undergo extensive unpacking with overexpression of DNA repair proteins supporting the idea of DNA damage contributing to the mechanism of the drug [37]. This finding may represent an additional advantage of $T$. dionisii that can even detect compounds that have been discarded due to their high IC50 values in previous tests using T. cruzi. It is well known that different lineages of T. cruzi display significant differences in drug sensitivity. Therefore, we recommend that after an initial screening of new compounds with T.dionisii those with higher potential shall be tested against strains representative of the various lineages.

The electron microscopy data also showed that the effects of the three compounds on $T$. dionisii structural organization were the same as described previously for $T$. cruzi [28]. These include the following features: (a) swelling in the mitochondrionkinetoplast complex, (b) alteration of the Golgi complex and (c) formation of autophagosomes characterized both by their morphological appearance on TEM and by LC3-B labeling as seen by immunofluorescence microscopy. These findings indicate parasite death due to an autophagic process as previously reported for T. cruzi [28].

In conclusion our results show that $T$. dionisii is an excellent model for initial screening of compounds to be subsequently tested against $T$. cruzi and due to these observation it is now incorporated into our platform of screening of compounds supported by the Ministry of Health and the National Research Council of Brazil.

\section{Acknowledgment}

The authors thank Luis Sergio Cordeiro Junior and Luzinete Silva for technical assistance. This work was supported by Conselho Nacional de Desenvolvimento Cientifico e Tecnológico (CNPq), Financiadora de Estudos e Projetos (FINEP) and Fundação Carlos Chagas Filho de Amparo à Pesquisa do Estado do Rio de Janeiro (Faperj).

\section{References}

1. WHO. 2017; Ref.: https://goo.gl/jbBZvZ

2. Rassi A Jr, Rassi A, Marcondes de Rezende J. American trypanosomiasis (Chagas disease). Infect Dis Clin North Am. 2012; 26: 275-291. Ref.: https://goo.gl/wM6cjx 
3. Rassi Jr A, Rassi A, Marin-Neto JA. Chagas heart disease: pathophysiologic mechanisms, prognostic factors and risk stratification. Mem Inst Oswaldo Cruz. 2009; 104: 152-158. Ref.: https://goo.gl/WGEr4U

4. Conners EE, Vinetz JM, Weeks JR, Brouwer KC. A global systematic review of Chagas disease prevalence among migrants. Acta Trop. 2016; 156: 68-78. Ref.: https://goo.gl/ZDcCyo

5. De Souza W, de Carvalho TM, Barrias ES. Review on Trypanosoma cruzi: Host Cell Interaction. Int J Cell Biol. 2010; pil: 295394. Ref.: https://goo.gl/94ZVUq

6. De Souza W. An introduction to the structural organization of parasitic protozoa. Curr Pharm Des. 2008; 14: 822-838. Ref.: https://goo.gl/avGpYF

7. Silva-Dos-Santos D, Barreto-de-Albuquerque J, Guerra B, Moreira OC, Berbert LR, et al. Unraveling Chagas disease transmission through the oral route: Gateways to Trypanosoma cruzi infection and target tissues. PLoS Negl Trop Dis. 2017; 11: e0005507. Ref.: https://goo.gl/bkrtcF

8. Barbosa Md, Ferreira JM, Arcanjo AR, Santana RA, Magalhães LK, et al. Chagas disease in the State of Amazonas: history, epidemiological evolution, risks of endemicity and future perspectives. Rev Soc Bras Med Trop. 2015; 48 Suppl 1: 27-33. Ref.: https://goo.gl/duyD6y

9. Pinto AY, Valente SA, Valente Vda C, Ferreira Jr AG, Coura JR. Acute phase of Chagas disease in the Brazilian Amazon region: study of 233 cases from Pará, Amapá and Maranhão observed between 1988 and 2005. Rev Soc Bras Med Trop. 2008; 41: 602-614. Ref.: https://goo.gl/yDccsD

10. Salomao K, Menna-Barreto RF, de Castro SL. Stairway to Heaven or Hell? Perspectives and Limitations of Chagas Disease Chemotherapy. Curr Top Med Chem. 2016; 16: 2266-2289. Ref.: https://goo.gl/GGBjmn

11. Sales Junior PA, Molina I, Fonseca Murta SM, Sánchez-Montalvá A, Salvador F, et al. Experimental and Clinical Treatment of Chagas Disease: A Review. Am J Trop Med Hyg. 2017; Ref.: https://goo.gl/3DzXuz

12. Ferraz ML, Gazzinelli RT, Alves RO, Urbina JA, Romanha AJ. The Anti-Trypanosoma cruzi activity of posaconazole in a murine model of acute Chagas' disease is less dependent on gamma interferon than that of benznidazole. Antimicrob Agents Chemother. 2007; 51: 1359-1364. Ref.: https://goo.gl/kL6XiB

13. Chirac $P$, Torreele E. Global framework on essential health R\&D. Lancet. 2006; 367: 1560-1561. Ref.: https://goo.gl/suzghZ

14. Khare $S$, Nagle AS, Biggart A, Lai $\mathrm{YH}$, Liang $\mathrm{F}$, et al. Proteasome inhibition for treatment of leishmaniasis, Chagas disease and sleeping sickness. Nature. 2016; 537: 229-233. Ref.: https://goo.gl/ffmP4v

15. Herwaldt BL. Laboratory-acquired parasitic infections from accidental exposures. Clin Microbiol Rev. 2001; 14: 659-688. Ref.: https://goo.gl/mXr1qW

16. Brener Z, Gazzinelli RT. Immunological control of Trypanosoma cruzi infection and pathogenesis of Chagas' disease. Int Arch Allergy Immunol. 1997; 114: 103-110. Ref.: https://goo.gl/vXxBkH

17. Hamilton PB, Lewis MD, Cruickshank C, Gaunt MW, Yeo M, et al. Identification and lineage genotyping of South American trypanosomes using fluorescent fragment length barcoding. Infect Genet Evol. 2011; 11: 44-51. Ref.: https://goo.gl/56Bn13

18. Maeda FY, Cortez C, Alves RM, Yoshida N. Mammalian cell invasion by closely related Trypanosoma species T. dionisii and T. cruzi. Acta Trop. 2012; 121: 141-147. Ref.: https://goo.gl/AcRZFo

19. Oliveira MP, Cortez M, Maeda FY, Fernandes MC, Haapalainen EF, et al. Unique behavior of Trypanosoma dionisii interacting with mammalian cells: invasion, intracellular growth, and nuclear localization. Acta Trop. 2009; 110: 65-74. Ref.: https://goo.gl/ev8k3E

20. Leslie M. Infectious diseases. Drug developers finally take aim at a neglected disease. Science. 2011; 333: 933-935. Ref.: https://goo.gl/yPHT3q

21. Gaborak M, Darling JL, Gutteridge W. Comparative drug sensitivities of culture forms of Trypanosoma cruzi and Trypanosoma dionisii. Nature. 1977; 268: 339-340. Ref.: https://goo.gl/3r4sww

22. Ministério da Saúde (BR), Secretaria de Vigilância em Saúde Ministério da Saúde. Consenso Brasileiro em doença de Chagas. Rev Soc Bras Med Trop. 2005; 38: 1-29.

23. Rassi A Jr, Rassi A, Little WC. Chagas' heart disease. Clin Cardiol. 2000; 23: 883-889. Ref.: https://goo.gl/kPbAis

24. Carmo AA, Rocha MO, Silva JL, lanni BM, Fernandes F, et al. Amiodarone and Trypanosoma cruzi parasitemia in patients with Chagas disease. Int J Cardiol. 2005; 189:182-184. Ref.: https://goo.gl/hFd5SC 
25. Benaim G, Sanders JM, Garcia-Marchán Y, Colina C, Lira R, et al. Amiodarone has intrinsic antiTrypanosoma cruzi activity and acts synergistically with posaconazole. J Med Chem. 2006; 49: 892899. Ref.: https://goo.gl/8uQfCk

26. Molina I, Salvador F, Sánchez-Montalvá A. The use of posaconazole against Chagas disease. Curr Opin Infect Dis. 2015; 28: 397-407. Ref.: https://goo.gl/2Na5yH

27. Diniz Lde F, Urbina JA, de Andrade IM, Mazzeti AL, Martins TA, et al. Benznidazole and posaconazole in experimental Chagas disease: positive interaction in concomitant and sequential treatments. PLoS Negl Trop Dis. 2013; 7: e2367. Ref.: https://goo.gl/PgaiQq

28. Veiga-Santos P, Barrias ES, Santos JF, de Barros Moreira TL, de Carvalho TM, et al. Effects of amiodarone and posaconazole on the growth and ultrastructure of Trypanosoma cruzi. Int $\mathrm{J}$ Antimicrob Agents. 2012; 40: 61-71. Ref.: https://goo.gl/NZkEZy

29. Morillo CA, Marin-Neto JA, Avezum A, Sosa-Estani S, Rassi A Jr. et al. Randomized trial of benznidazole for chronic Chagas' cardiomyopathy. N Engl J Med. 2015; 373: 1295-1306. Ref.: https://goo.gl/qxwUHE

30. Camargo EP. Growth and differentiation in Trypanosoma cruzi. I. Origin of metacyclic trypanosomes in liquid media. Rev Inst Med Trop Sao Paulo. 1964; 6: 93-100. Ref.: https://goo.gl/5ZxiQk

31. Baker JR, Selden LF. Trypanosoma (Schizotrypanum) dionisii: influence of mouse peritoneal macrophages and calf sera on extracellular growth in vitro at $37^{\circ} \mathrm{C}$. J Gen Microbiol 1978; 106: 27 32. Ref.: https://goo.gl/fhJGzB

32. Lepesheva GI, Villalta F, Waterman MR. Targeting Trypanosoma cruzi sterol 14a-demethylase (CYP51). Adv Parasitol 2011; 75: 65-87. Ref.: https://goo.gl/3RaB6B

33. Petrikkos G, Skiada A. Recent advances in antifungal chemotherapy. Int J Antimicrob 2007; 30: 108117. Ref.: https://goo.gl/WZRMd5

34. Benaim B, Garcia CR. Targeting calcium homeostasis as the therapy of Chagas' disease and leishmaniasis - a review. Trop Biomed. 2011; 28: 471-481. Ref.: https://goo.gl/zYfTC6

35. Maya JD, Cassels BK, Iturriaga-Vasquez P, Ferreira J, Faundez M, et al. Mode of action of natural and synthetic drugs against Trypanosoma cruzi and their interaction with the mammalian host. Comp Biochem Physiol A Mol Integr Physiol. 2007; 146: 601-620. Ref.: https://goo.gl/1DhW5x

36. Romanha AJ, Alves RO, Murta SM, Silva JS, Ropert C, et al. Experimental chemotherapy against Trypanosoma cruzi infection: essential role of endogenous interferon-gamma in mediating parasitologic cure. J Infect Dis. 2002; 186: 823-828. Ref.: https://goo.gl/XTVoMZ

37. Rajao MA, Furtado C, Alves CL, Passos-Silva DG, de Moura MB, et al. Unveiling benznidazole's mechanism of action through overexpression of DNA repair proteins in Trypanosoma cruzi. Environ Mol Mutagen. 2014; 55: 309-321. Ref.: https://goo.gl/44oUw6 